\title{
Efficient QTL detection for nonhost resistance in wild lettuce: backcross inbred lines versus $F_{2}$ population
}

\author{
M. J. W. Jeuken · K. Pelgrom · P. Stam • \\ P. Lindhout
}

Received: 24 October 2007 / Accepted: 12 January 2008/Published online: 5 February 2008

(C) The Author(s) 2008

\begin{abstract}
In plants, several population types $\left[\mathrm{F}_{2}\right.$, recombinant inbred lines, backcross inbred lines (BILs), etc.] are used for quantitative trait locus (QTL) analyses. However, dissection of the trait of interest and subsequent confirmation by introgression of QTLs for breeding purposes has not been as successful as that predicted from theoretical calculations. More practical knowledge of different QTL mapping approaches is needed. In this recent study, we describe the detection and mapping of quantitative resistances to downy mildew in a set of 29 BILs of cultivated lettuce (L. sativa) containing genome segments introgressed from wild lettuce (L. saligna). Introgression regions that are associated with quantitative resistance are considered to harbor a QTL. Furthermore, we compare this with results from an already existing $\mathrm{F}_{2}$ population derived from the same parents. We identified six QTLs in our BIL approach compared to only three in the $\mathrm{F}_{2}$ approach, while there were two QTLs in common. We performed a simulation study based on our actual data to help us interpret them. This revealed that two newly detected QTLs in the BILs had gone unnoticed in the $F_{2}$, due to a combination of recessiveness of the trait and skewed segregation, causing a deficit of the wild species alleles. This study clearly illustrates the added value of extended genetic studies on two
\end{abstract}

Communicated by M Sillanpää.

M. J. W. Jeuken · K. Pelgrom · P. Stam · P. Lindhout Laboratory of Plant Breeding, Wageningen University, PO Box 386, 6700 AJ Wageningen, The Netherlands

M. J. W. Jeuken ( $\square)$

Laboratory of Plant Breeding, Wageningen University, Droevendaalsesteeg 1, 6708 PB Wageningen, The Netherlands e-mail: marieke.jeuken@wur.nl different population types (BILs and $\mathrm{F}_{2}$ ) to dissect complex genetic traits.

\section{Introduction}

In the search for more durable disease resistance in plants, scientists and breeders are now also focusing on exploring alternative resistances like quantitative resistance. Quantitative resistance exhibits properties that differ from the widely used qualitative resistances, which are explained by $\mathrm{R}$ genes that interact with Avr-genes (Lindhout 2002). Quantitative resistance is a complex trait, as it exhibits a continuous phenotypic variation and is mostly under polygenic control [quantitative trait loci (QTLs)]. Breeders are interested in detecting and introgression of QTLs for resistance and also QTLs for other quantitative traits like quality and yield (Varshney et al. 2006). However, the introgression of QTLs (not specifically QTLs for resistance) by markerassisted selection (MAS) for breeding purposes has often shown inconsistent results and has not been as successful as that predicted from theoretical calculations (Schön et al. 2004). This is mainly caused by the lack of efficient and accurate QTL mapping approaches that depend on some critical key factors like population type, population size, marker density, the quality of the phenotypic evaluation, environment, number of QTLs, the magnitude of the effect, the inheritance of the QTL (dominant, recessive or additive), the genotype $\times$ environment interaction and the epistatic interaction between loci.

Several theoretical studies show the importance of the population size on the power of QTL detection (Beavis 1994, 1998; Van Ooijen 1999). For example, Beavis showed that when using a population of 100 plants, the 
chance of detecting a QTL with an explained phenotypic effect of $<10 \%$ was only $3 \%$, and the magnitude of estimated effect was 10-fold inflated. Furthermore, increasing the population size to 500 plants improved the accuracy considerably, and in a population of 1,000 plants the magnitude of the phenotypic effect was close to the actual value. These theoretical studies have been supported by field experiments in which an increase of sample sizes resulted in an increase in the number of detected QTLs explaining the trait (Schön et al. 2004; Vales et al. 2005).

Often, phenotypic evaluations and marker screenings on large population sizes of $>500$ are not used, as they are too laborious and costly. This especially holds true for wide crosses where the chance to obtain vital progenies is often very low.

Alternative approaches aiming to improve QTL mapping efficiency without large population sizes have been developed. Examples are linkage disequilibrium (LD) mapping (Flint-Garcia et al. 2003; Gupta et al. 2005; Kraakman et al. 2004; Yu et al. 2006), meta-analyses (Arcade et al. 2004; Guo et al. 2006) or QTL mapping on selected informative genotypes only (Peleman et al. 2005). Alternatively, a limited set of inbred lines [near-isogenic lines (NILs) or backcross inbred lines (BILs) or advanced backcross (AB) lines] can be used instead of a large $\mathrm{F}_{2}$ or recombinant inbred line (RIL) population (Keurentjes et al. 2007; Tanksley and Nelson 1996; Zamir 2001). Since such lines are homozygous, numerous genetically identical plants can be evaluated, which will increase the accuracy of phenotyping without increasing the efforts of genotyping. In addition, this also allows extended genotypes $\times$ environment studies.

We choose this "Inbred Line" strategy for our research topic "Genetic dissection of the non-host resistance of wild lettuce" because of the above mentioned advantages and because the genetically wide cross between resistant wild lettuce (L. saligna) and the susceptible lettuce ( $L$. sativa) limited the generation of a large population of vital and (self-)fertile $\mathrm{F}_{2}$ plants or even RILs.

We repeatedly backcrossed the parental L. saligna $\times L$. sativa-cross with the $L$. sativa parent until the $\mathrm{BC}_{4}$ generation. The $\mathrm{BC}_{4} \mathrm{~S}_{1}$ population and later generations, obtained by selfing, were screened by MAS, and this resulted in a set of 29 BILs. Each BIL contains one introgression of L. saligna, harboring on average $4 \%$ of the L. saligna genome. The set of 29 BILs together cover $96 \%$ of the L. saligna genome (Jeuken and Lindhout 2004).

In the present study, we describe the detection and mapping of QTLs for downy mildew resistance in this set of 29 BILs. Furthermore, we estimate the magnitude of the QTL effects and the inheritance of QTLs.

Previously, we have executed a QTL mapping study on a small $F_{2}$ population of 126 plants (based on the identical parental cross; Jeuken and Lindhout 2002). This now allows us to compare the two studies with regard to detection of the number and the position of the QTLs and to compare the actual efficiency of QTL mapping by both approaches. Because of the diminished genetic noise and the increased accuracy of phenotyping in the set of BILs, it was expected that this approach would result in the identification of more QTLs than when using the $F_{2}$ approach. Only if positive, trans-acting epistatic interactions are involved might a QTL be identified in an $\mathrm{F}_{2}$ population but remain hidden in a set of BILs.

As the present study represents the results of a single experiment, which was a sample from many possible experimental results, we have performed a simulation study to assess the probability that a QTL is detected in the $\mathrm{F}_{2}$ and in the BILs. For a given set of values of biological and experimental parameters like population size, true QTL effect, marker density, skewness of segregation and accuracy of phenotypic evaluation, 50,000 replicate $\mathrm{F}_{2}$ populations or sets of BILs were sampled. By varying the parameter values, we were able to quantitatively study their influence on the chance of detecting a QTL. The "default" values of these parameters were based on the actual results of the mapping studies of the $F_{2}$ and the BILs. These simulations help us to interpret mapping results of real data and to see the impact of segregation distortion on QTL detection power.

In the present study, the experimental QTL mapping approaches by using an $\mathrm{F}_{2}$ population and a set of BILs originating from the same parental cross are compared with each other and with in silico results. This state of affairs represents a unique combination of experimental and theoretical approaches to verify QTL mapping efficiencies, which to our knowledge, is a novelty in this research area. Although the actual results of the present study are valid for the lettuce-downy mildew model, our method can readily be applied to other quantitative traits and other plant species, and therefore, is of quite general value for developing QTL mapping strategies.

\section{Materials and methods}

Summary of materials, methods and results on the $F_{2}$ population

The $F_{2}$ plant material, genetic linkage map and QTL analyses have been described earlier in Jeuken et al. (2001) and Jeuken and Lindhout (2002).

\section{Summary}

The $F_{2}$ population consisted of 126 plants and was genotyped with about 500 AFLP markers. A genetic linkage 
map was constructed with a random marker distribution covering all nine chromosomes. The average marker spacing was $1.8 \mathrm{cM}$, and $65 \%$ of the marker distances lied between 1 and $8 \mathrm{cM}$ (Jeuken et al. 2001).

Four disease tests on leaf discs of $F_{2}$ plants infected by B. lactucae races $\mathrm{Bl}: 14$ and $\mathrm{Bl}: 16$ were executed. Per disease test, six leaf discs per $F_{2}$ plant were scored for infection severity. Finally, a QTL analysis was executed with MapQTL software (Van Ooijen 2004), performing a Kruskal-Wallis test, simple Interval Mapping and approximate multiple QTL mapping (MQM) with all genotypic (markers) and phenotypic (disease test) data. This resulted in the detection of one race-specific $R$-genelike resistance designated $R 39$ and three race-nonspecific QTLs designated $R b q 1, R b q 2$ and $R b q 3$. These three QTLs were located at Chromosomes 7, 1 and 9, had a peak LOD score of 7, 3 and 4 and had a proportion of explained variance of 26, 13 and 12\%, respectively (Jeuken and Lindhout 2002). The heritability of resistance to B. lactucae races $\mathrm{Bl}: 14$ and $\mathrm{Bl}: 16$ was high $(>0.8)$ in the $\mathrm{F}_{2}$ population, and the three QTLs explained only $51 \%$ of the quantitative resistance. This indicated that other resistance QTLs might segregate in the $\mathrm{F}_{2}$ population.

\section{Plant material}

\section{Tests on the set of 29 BILs}

A set of 29 BILs plus the two parental lines were used for disease tests. The parental lines are the cultivar $L$. sativa cv. Olof, which is the recurrent parent that does not harbor any known $D m$ gene (downy mildew $\mathrm{R}$ gene) and is therefore susceptible to all described B. lactucae races, and L. saligna CGN05271 as the nonhost donor parent.

The set of 29 BILs has been developed earlier and details about the nomenclature, development or the genotypes of the set of BILs are described in (Jeuken and Lindhout 2004). Summarized, this set of BILs was genotyped with more than 700 DNA markers (AFLP, ESTs and SSRs) and covers $96 \%$ of the L. saligna genome. On average, each BIL contained one homozygous introgression fragment of the wild species of $33 \mathrm{cM}$ in a $L$. sativa background ( $=4 \%$ of the L. saligna genome). An exception is four BILs that remain heterozygous for their introgression. The nomenclature of the BILs refers to the location of the introgression, for example, BIL4.3 stands for the third introgression on Chromosome 4.

Modifications on the description of BILs in (Jeuken and Lindhout 2004) are as follows: Chromosome 8 of $L$. saligna is completely represented by four BILs (since an AFLP-marker E48M59-197 was detected that showed an overlap between the introgression region of BIL8.2 and
BIL8.3); BIL1.1 is now homozygous L. saligna for the whole introgression region. A "doubleBIL" was added to the set of 28 BILs to improve resolution on Chromosome 7, designated "doubleBIL7.1," which has one large and one small homozygous $L$. saligna introgression region; one on Chromosome 7 from 25 to $40 \mathrm{cM}$ and one on Chromosome 1 from 76 to $87 \mathrm{cM}$. These 29 BILs represent 53 Bins. (Bins are arbitrarily-defined segments delimited by two fixed core markers. A Bin includes all loci from the leftmost or top core marker to the next core marker.)

A "preBIL" is a line that has a single heterozygous $L$. saligna introgression, while a "BIL" carries a homozygous introgression from L. saligna. Plants were grown in the greenhouse in a randomized block design.

\section{Disease test}

\section{Tests on the set of 29 BILs}

Six disease tests with two downy mildew races were performed on selections of the set of 29 BILs between 2001 and 2006. The first and the fifth tests were performed with B. lactucae race $\mathrm{Bl}: 16$ and the other four tests with $\mathrm{Bl}: 14$. For practical reasons, we performed six incomplete tests with twenty BILs instead of using complete tests with all 29 BILs. In each of the six incomplete tests, a different combination of eleven BILs plus the same nine "reference BILs" plus the two parental lines were included. The reference BILs were selected after the first two disease tests. All BILs that showed significantly lower infection levels were selected and a few BILs with a similar infection level as the susceptible parental line were selected. The reference BILs are BIL1.2, 2.2, 4.2, 4.4, 5.1, 6.3, 8.2, 8.4 and 9.2. The other 20 BILs were rotated between the six incomplete tests and were tested in at least three out of six tests. In each disease test, seven plants were analyzed per BIL and per control parent line, with four evaluated leaf discs per plant. The infection severity level of each BIL was compared with that of the susceptible recurrent parent L. sativa Olof and the other BILs. A BIL was considered quantitatively resistant if its infection severity level was significantly $(\alpha=0.05)$ lower than that of $L$. sativa Olof. A BIL that showed quantitative resistance was considered to harbor a QTL at the introgression region.

\section{Inheritance tests for BILs with resistance QTLs}

A selected preBIL population of twenty plants segregating for the introgression and four or five plants of the corresponding resistant BIL progeny were tested for resistance to two B. lactucae races. The twenty plants per preBIL 
were genotyped with codominant EST-markers based on lettuce EST sequences, one SSR marker and one phenotypic marker (Fig. 1, Table 1). DNA was extracted according to Jeuken and Lindhout (2004). Amplification of PCR-markers was performed in $25 \mu \mathrm{l}$ PCR reactions with $10 \mathrm{ng}$ DNA, $0.5 \mathrm{U}$ Taq polymerase, $10 \mathrm{pmol}$ of both primers, $2.5 \mu \mathrm{l} 10 \times$ reaction buffer and $5 \mathrm{mM}$ of all four dNTPs. The following PCR program was used: 2 ' $94^{\circ} \mathrm{C}$, $30 “ 94^{\circ} \mathrm{C}, 30 " 52^{\circ} \mathrm{C}, 1{ }^{\prime} 72^{\circ} \mathrm{C}, 2$ ' $72^{\circ} \mathrm{C}$. Steps $2-4$ were repeated for 40 cycles. For cleaved amplified polymorphic sequence digestion, we added $0.1 \mathrm{U}$ restriction enzyme and $3 \mu \mathrm{l}$ specific enzyme buffer and incubated for at least $3 \mathrm{~h}$ at $37^{\circ} \mathrm{C}$. PCR products were run on $1-2 \%$ agarose gels to separate products with larger size differences. Each introgression was specified by at least two markers that were less than $5 \mathrm{cM}$ away from the ends of the introgression. Only BIL8.2 and BIL4.2 had a marker further away from the top end at 7 and $14 \mathrm{cM}$, respectively. The SSR marker LsB104 is described in (Van de Wiel et al. 1999). EST markers were developed from lettuce EST sequences by the Compositae Genome Project (http://cgpdb.ucdavis.edu); information for these markers is available from the project web site (http://cgpdb.ucdavis.edu/database/genome_ viewer/viewer/). The primer sequences for each marker are listed in Table 1. Marker loci were classified in one of the following three genotype classes: homozygous $L$. sativa (code "A"), heterozygous (code " $\mathrm{H}$ ") or homozygous $L$. saligna (code "B"). Each genotype class was represented by five plants per preBIL population. Genotypes that showed a recombination event within the introgression region were excluded from analyses. If the genotype class "homozygous L. saligna" contained fewer than five plants, it was filled up with plants of their counterpart BILs to equalize numbers of plants in the three genotype classes and to compensate for expected preference of $L$. sativa alleles for preBIL8.2 and preBIL6.3 population.

\section{Protocol disease test}

Disease tests were performed according to Jeuken and Lindhout (2002) with minor modifications. Nine to eleven days after inoculation, the leaf discs were assessed for growth of sporangiophores. Infection severity was scored visually as the percentage of leaf area covered with sporangiophores.

\section{B. lactucae races}

The maintenance and the inoculation of B. lactucae were described in (Jeuken and Lindhout 2002). We used identical B. lactucae races for this study. However, the names of these races (NL14 and NL16) are now designated according the International Bremia Evaluation Board as races $\mathrm{Bl}: 14$ and $\mathrm{Bl}: 16$.

\section{Data analysis}

\section{Tests on the set of 29 BILs}

All infection severity scores from the disease tests were arcsine square-root-transformed to improve the
Fig. 1 The position of EST and CAPs markers on introgressions of BIL2.2, 4.2, 6.3, 8.2 and 9.2 are shown per chromosome. Black boxes represent introgressions on BILs. Restriction enzymes are shown with CAPS marker if necessary to reveal a polymorphism. P92 is typical leaf phenotype from BIL9.2

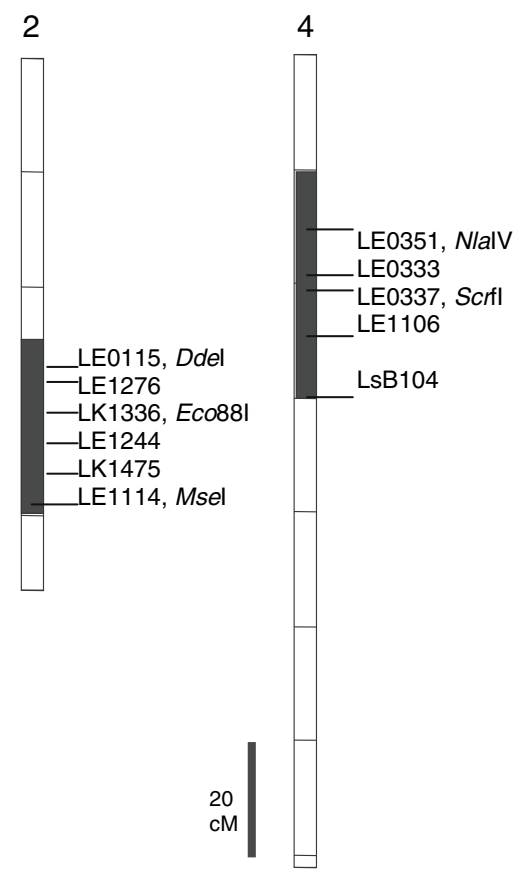

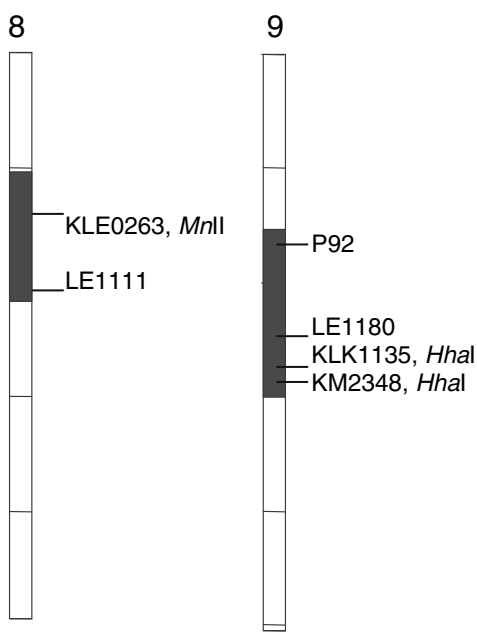


Table 1 Primer sequences of EST markers

\begin{tabular}{lll}
\hline Marker name & Primer forward & Primer reverse \\
\hline LE0115 & ACTGCTCCACTACCCACCAC & CGACAAGGCTGACATCGTAA \\
LE1276 & TTTGGGTTCCTTCAGTTTGC & CACAGTTTGGGATGAACACG \\
LK1336 & TGAGGAGTCCATGGATACGG & CGATGCAACAGCATGGATAC \\
LE1244 & CATCCGCTTCCTCTTCAGTC & ACGAGCACCTGCATCTACAA \\
LK1475 & GGAGTTCAGGGCCTCTGTC & CCGATTCTGCGGTTATCTTC \\
LE1114 & CAAGAGGTGAATGGGAAGGA & TACCACACAAACAAGCGGAA \\
LE0351 & GAATATGCGGCGGAGATAAG & AATCACATGAATGGATGCAAA \\
LE0333 & GGACCGGGTTTTTAAGTCGT & TTTCTCTGTATATATGCAATCTCCATT \\
LE0337 & CCATGGCTAAAAAGCAAACC & ACATTAGCCAAGCGACAACA \\
LE1106 & TGATTATGGAGGCGAAGAGG & CACAAAGATTCATTACTTGCCATC \\
LE1126 & CTTTGCTCCAATTCCTCTCG & AATGCCATAGTGAAGCTGGG \\
M7120 & ACAGCAACAGCCGACCG & CGCACATTATTCGGCTCAAA \\
LE1211 & CGGGTGATTACATCGGCTAT & CGCAACCAACCAAATTTACC \\
KLE0263 & CAACCTCACCGGAGTTTTGT & GCCGGAAAGTTTGTTGTTGT \\
LE1111 & AATTCACTCCACCACCGAAG & CTACGTCAGTGCCTATGCCA \\
KLK1135 & TAAACTTCGGGACGAACCAC & GCCAAAATGCGAAAGTTGC \\
LE1180 & TTGAGGTTTATGGACCCGAG & CAAGCGCGCACTGATAGTAA \\
KM2348 & TAAACTTCGGGACGAACCAC & GCCAAAATGCGAAAGTTGC \\
LE0115 & ACTGCTCCACTACCCACCAC & CGACAAGGCTGACATCGTAA \\
LE1276 & TTTGGGTTCCTTCAGTTTGC & CACAGTTTGGGATGAACACG \\
\hline
\end{tabular}

homogeneity of residual variance before further calculations were performed. The infection severity level of one plant was calculated as the average of four leaf discs per plants per disease test.

Differences in mean infection severity levels of BILs over six tests (seven plants per line per test) were analyzed by a mixed-model procedure with "experiment" and "genotype" as fixed factors (Patterson and Thompson 1971). Tukey HSD (honestly significantly difference) multiple comparison procedure was used as a post hoc test to determine significant differences among 29 BILs and their parental lines $(\alpha=0.05$ and $\alpha=0.01)$. In the Tukey HSD procedure, the error rate that is controlled is an experimentwise error rate over all pairwise comparisons.

As different seasons might influence plant conditions and resistance levels, the similarity between the six disease tests was measured by a two-way ANOVA with replication on the nine reference BILs plus L. sativa Olof. Race specificity was measured by two-way ANOVA with replication between disease tests 1 and 2 and 5 and 6 with $B$. lactucae race $\mathrm{Bl}: 14$ and race $\mathrm{Bl}: 16$ on separate leaf discs from the same set of plants.

\section{Inheritance tests for QTLs}

Deviations of normal segregation ratios (skewness) of the introgressions of the preBIL populations in the three genotype classes (homozygous L. sativa, heterozygous, homozygous L. saligna) were tested by using a chi-square test.

In each preBIL population, the infection severity levels were tested per genotype class by one-way ANOVA and the means were compared by Tukey HSD test.

In silico QTL detection experiments

We have written a simulation program, which mimics QTL detection in an $F_{2}$ population and in a set of BILs. The purpose of the in silico experiments carried out with this program was to investigate the role of several factors that determine the probability of detection of a QTL of given effect. The program calculates the probability of detecting a QTL in an $\mathrm{F}_{2}$ population by performing an approximate QTL mapping procedure on numerous (preferably $>10,000$ ) samples of $F_{2}$ populations and BILs. The proportion of simulation runs in which the QTL is detected is then taken as the detection probability. When running the program, the following variables can be set: average score (phenotypic value) of the presumed QTL (i.e. the QTL effect), $F_{2}$ population size, genetic distance between QTL and nearest marker (designated as "marker density"), segregation ratio at this marker locus (designated as "skewness"), number of QTLs (of equal effect) underlying the trait, total phenotypic variance in the $F_{2}$ population and number of observations per $\mathrm{F}_{2}$ plant. 
For simulation of the BILs, we used a two-sample $t$ test for each BIL to be tested against the control (L. sativa cv. Olof), applying a Bonferroni correction for multiple testing. Mean values for BILs and control were the same as those used in the corresponding $\mathrm{F}_{2}$ experiment. For the set of BILs, the following variables can be set: number of BILs to be tested, number of plants per line and number of samples per plant. A detailed description of the simulation program is given in Appendix A.

As a "standard configuration" to which all other simulation runs could be compared, we used parameter settings that correspond to our actual $\mathrm{F}_{2}$ population. Thus, by changing one of the parameter values at a time (e.g. population size, segregation ratio, number of observations per plant, etc.), we were able to investigate the influence of these parameter values on the chance of detection in an actual experiment and how detection probability decreases or increases as one moves away from the "standard configuration."

The standard parameter settings were as follows. Genotypic values of the presumed QTL were based on the average phenotypic scores at loci $R b q 1, R b q 2$ and $R b q 3$, obtained from the actual QTL analysis on the $\mathrm{F}_{2}$ population tested with $B$. lactucae race $\mathrm{Bl}: 14$. These values are as follows:

$\begin{array}{ll}\text { Homozygous L. sativa: } & 2.5 \\ \text { Homozygous L. saligna: } & 1.7 \\ \text { Heterozygous: } & 2.5, \text { if QTL is dominant } \\ \text { Heterozygous: } & 1.7, \text { if QTL is recessive }\end{array}$

From the same QTL analyses on our $\mathrm{F}_{2}$, the total phenotypic variance was taken to be 1.3 . The standard segregation ratio at the marker locus was 1:2:1 (MM:Mm:mm), and the standard number of observations per plant was 6 . For the standard genetic distance between QTL and nearest marker, we choose $5 \mathrm{cM}$, a representative value, since $65 \%$ of the marker distances were between 1 and $8 \mathrm{cM}$. The default number of segregating QTLs was taken to be 7 , i.e. the number of QTLs detected altogether in the $F_{2}$ population and the set of BILs.

Although the simulation program has an option to mimic interval mapping (IM) as well as composite interval mapping (CIM), we only used IM. The reason for this is that mimicking CIM requires knowledge of the proportion of the variance that is explained by markers near the background QTLs, a quantity that cannot be known beforehand. The number of simulation runs was 50,000 for each configuration of parameter values. The program was written in $\mathrm{C}++$; an executable, with graphical interface running under OS Windows, is available from P.S. upon request.

\section{Results}

A summary of the former $F_{2}$ analyses and results is presented in the "Materials and methods."

\section{Disease tests on a set of BILs}

The objectives of these experiments were to detect quantitative resistance loci (QTLs) in the set of BILs and to quantify their resistance levels. The infection severity of the parental lines in six tests was always $0 \%$ for L. saligna and ranged from 80 to $94 \%$ for L. sativa Olof, indicating a high reproducibility between the six tests. Similarly, the infection severity levels of the nine reference BILs were similar in all six tests.

A multiple comparison statistical test classified the 29 BILs in groups based on the infection severity levels over six tests (Fig. 2). Twenty-two BILs had a similar infection level as the susceptible parent $L$. sativa Olof and were

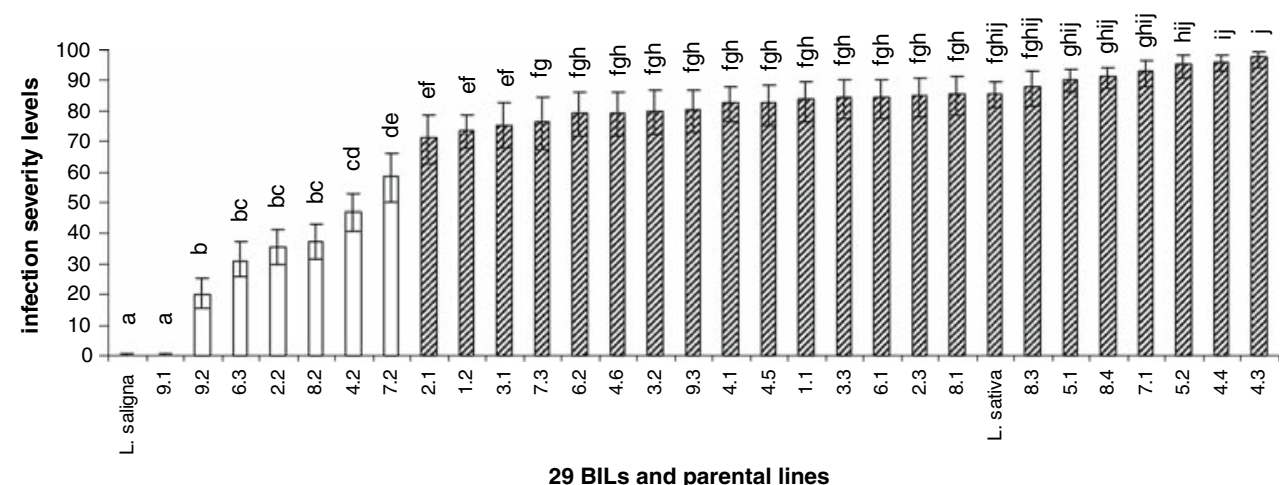

Fig. 2 Infection severity levels of twenty-nine BILs and parental lines over six disease tests with B. lactucae are shown. Ninety-five percent confidence intervals are shown by lines on top of the bars. Letters in common indicate that the values are not significantly different ( $\alpha=0.01$, Tukey HSD procedure). Striped bars represent lines not significantly different from $L$. sativa Olof. White bars represent lines that are significantly different from $L$. sativa Olof 
designated "susceptible." Six BILs were significantly different from the susceptible parent and were designated as "quantitatively resistant" $(\alpha=0.05$ and 0.01$)$. The quantitatively resistant group consists of BILs 9.2, 6.3, 2.2, 8.2, 4.2 and 7.2. Their infection severity levels ranged from 20 to $58 \%$ (Fig. 2). The susceptible group consists of the other twenty-two BILs and their infection levels ranged from 71 to $98 \%$. Line preBIL9.1 could not be classified in one of these groups, as it segregated into plants that were as susceptible as $L$. sativa Olof and plants that showed no infection. No race-specific interaction was detected for any of the BILs.

From the group of six quantitatively resistant lines, preBIL7.2 and BIL9.2 carry an introgression in which a QTL has been mapped previously (Jeuken and Lindhout 2002). The introgression region of preBIL7.2 coincides with $R b q 1$, and the introgression region of BIL9.2 corresponds with $R b q 3$. The introgression regions of BIL8.2, BIL 2.2, BIL6.3 and BIL4.2 do not carry known QTLs, and their detected quantitative resistances, which are considered as QTLs, are successively numbered from $R b q 4$ to Rbq 7 .

PreBIL9.1 differed clearly from all other lines. PreBIL9.1 is heterozygous for its introgression region that contains the locus of $R 39$, a race-specific R-gene detected in the $F_{2}$ population (Jeuken and Lindhout 2002). Without $R 39$, the plants were as susceptible as $L$. sativa Olof, while the presence of $R 39$ was associated with complete resistance to $B$. lactucae $\mathrm{Bl}: 16$ and $\mathrm{Bl}: 14$. An autonecrosis phenotype was exhibited by preBIL9.1 plants with $R 39$.

Disease tests to measure inheritance of five QTLs for resistance

We measured the size of the allelic effect of $R b q 3$ through $R b q 7$ in populations of twenty plants segregating for the corresponding Rbqs. To this end, five segregating populations ("pre-BILs") were used that were the ancestors of the corresponding BILs. In each of these preBIL populations, the genotype class "homozygous $L$. saligna" showed the expected low infection severity level that was significantly different from the two other genotype classes ("heterozygous" and "homozygous L. sativa") that were similar to the susceptible cv. Olof (Fig. 3). Consequently, Rbq3 through $R b q 7$ are recessive and are designated $r b q 3$ through $r b q 7$ according to the $B$. lactucae resistance nomenclature. The locations of the seven QTLs and R39 do not coincide with known R-gene clusters in the lettuce genome, except for $R b q 2$ and $r b q 4$, which show some overlap with Dm5/8 and Dm10 on Chromosome 1 and Dm13 on Chromosome 8, respectively (Fig. 4). The segregation of four of the preBIL populations was not

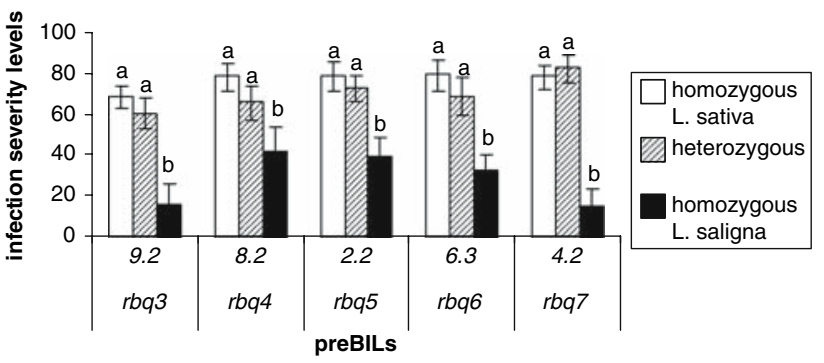

Fig. 3 The allelic effects of five QTLs were measured in a disease test with $B$. lactucae Bl:14 on preBIL populations. Infection severity levels and $95 \%$ confidence intervals are shown per QTL per genotype class that was represented by five plants. Letters in common within preBIL populations indicate that the values for genotype classes are not significantly different $(\alpha=0.05$, Tukey HSD procedure)

significantly different $(\alpha=0.05)$ from a monogenic $(1: 2: 1)$ segregation, while preBIL population 8.2 showed a 10:8:1 segregation, indicating skewed inheritance.

Sensitivity test for QTL detection in $\mathrm{F}_{2}$ and in BILs

Since we identified six QTLs in our BIL QTL mapping approach compared to only three in an $F_{2}$ mapping approach (with two QTLs in common), we wondered whether this result could be expected given the experimental conditions. Therefore, we designed a genetic model with seven QTLs and their allelic effects as estimated from the disease tests on the 29 BILs and the $F_{2}$ population. Subsequently, we estimated the chance of identifying a QTL in the $\mathrm{F}_{2}$ and in the BIL mapping approach given the experimental conditions used (marker density, population size and skewness; see "Materials and methods").

As a result, the average chance for detecting any of the seven QTLs in this $\mathrm{F}_{2}$ population detection was 0.7 (Fig. 5) and in the BILs was 0.98 (Table 2, last column). This model allowed us to estimate the effect of changing the experimental conditions used in the $\mathrm{F}_{2}$. For instance, the parameters that caused the most important effect on the detection chance were " $F_{2}$ population size" and "number of QTLs explaining the trait." As the density of the genetic map was already quite high, the marker distance had the least influence on the detection chance. Surprisingly, the segregation ratio also had an important effect on the chance of detecting a QTL. For example, when the frequency for the recessive genotype (homozygous L. saligna) varied from 0.25 to 0.06 , the QTL detection chance decreased from 0.70 to 0.17 . In contrast, when the frequency of a dominant QTL varied similarly, the detection chance increased only from 0.70 to 0.84 (Fig. 5). For both recessive and dominant QTLs, the decrease in detection chance due to skewness towards a certain parental genotype was much stronger than the increase of the detection chance 
Fig. 4 The position of QTLs and $R 39$ compared to known resistance gene clusters in lettuce according to Kesseli et al. (1994), Truco et al. (2007) and http://cgpdb.ucdavis.edu/
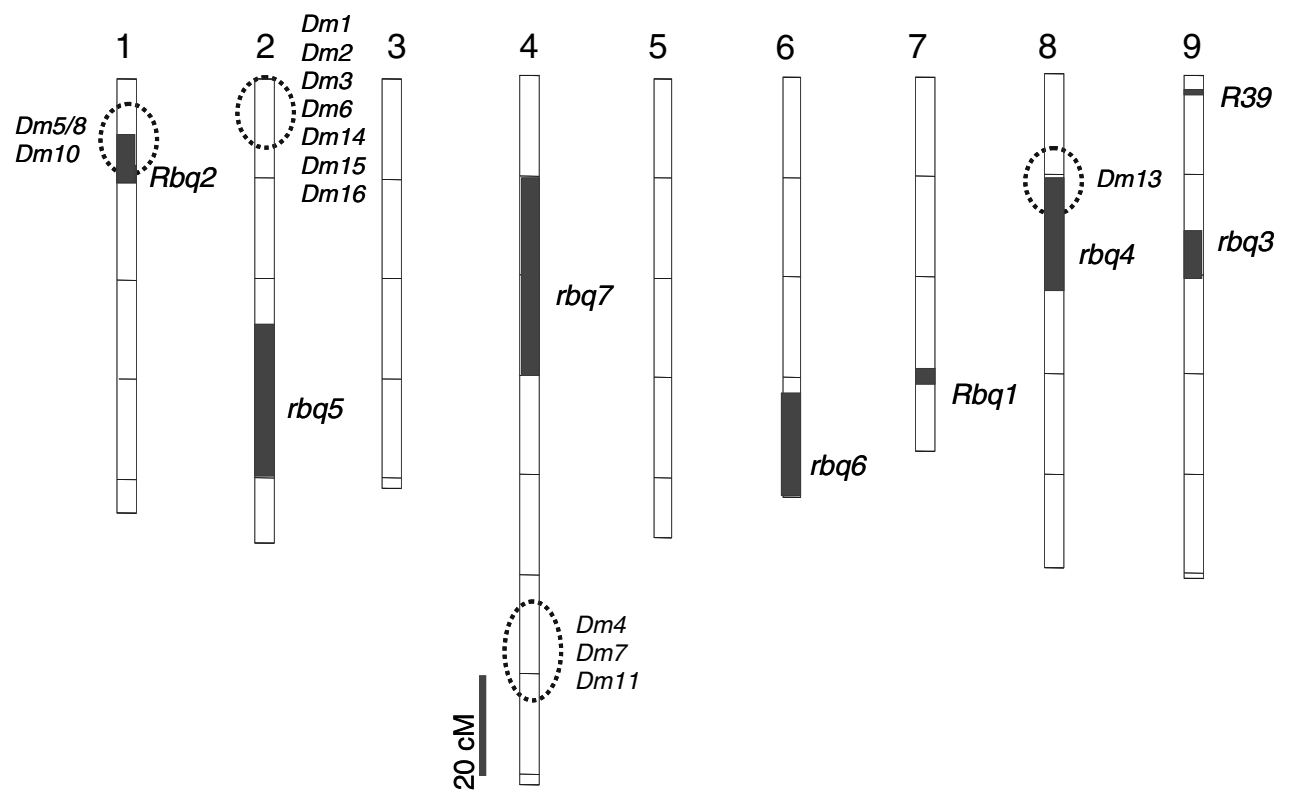

with skewness of the same value but in favor of the other parental genotype (Fig. 5).

Detection chances for the observed QTLs

Up to now, we did not differentiate between the QTLs in the sensitivity studies. However, we had real data on each of the QTLs, which allowed us to estimate the chance of identifying each QTL individually. This was based on three variables: (1) the inheritance (allelic effect), (2) the skewness and (3) the marker density (Table 2). Remarkably, by performing these simulation studies, we could explain the results of the $\mathrm{F}_{2}$ mapping approach very well. The detection chances were highest $(0.81-0.91)$ for the three QTLs detected in the $\mathrm{F}_{2}$ (Rbq1, Rbq2 and $R b q 3$ ), while the detection chance of the other QTLs was lower to extremely low (0.15-0.79). These extremely low detection chances were a result of the recessive character of the QTLs in combination with skewness against $L$. saligna alleles.

To have a detection chance of 0.8 for $r b q 3, r b q 4$ and $r b q 6$ with the same values for skewness, upwards of 250 to $550 \mathrm{~F}_{2}$ plants should have been needed for a detection chance of $95 \%$ (Fig. 6). This illustrates very well the great effect of distorted segregations on QTL detection chances. Recessive QTLs from the wild species became almost undetectable when they are located in a region with skewness against the wild species alleles.

With the size of the QTL effects observed in our experiment the detection chance in BILs was invariably high (0.98, last column in Table 2). Nevertheless, this

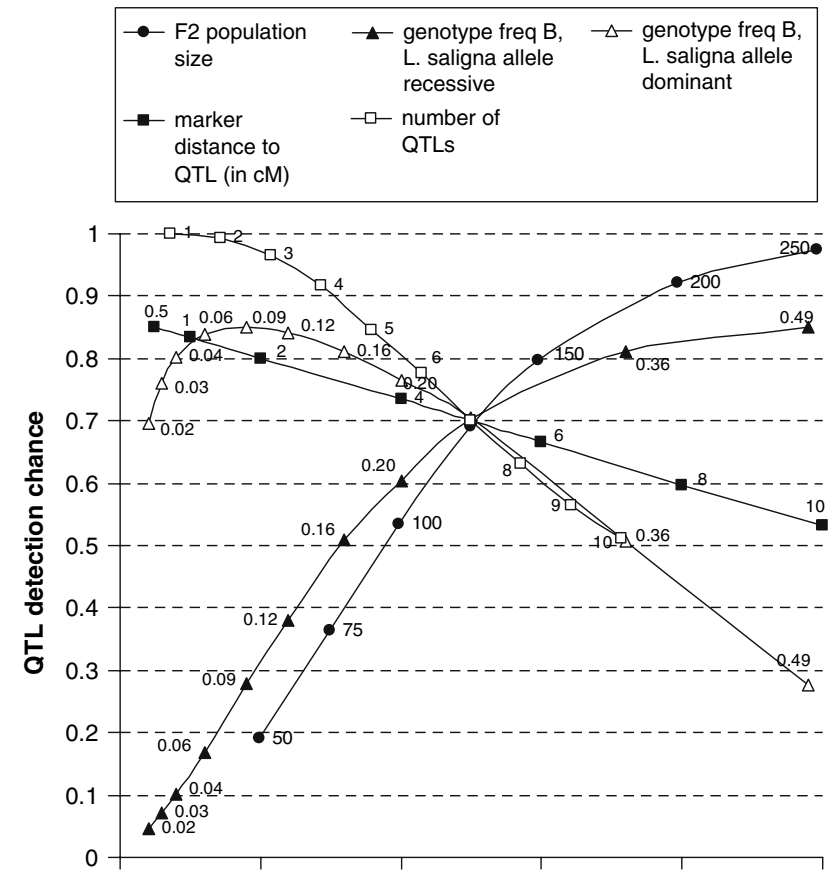

Fig. 5 Simulation studies show the chance of QTL detection in an $\mathrm{F}_{2}$ population. The starting point is an $\mathrm{F}_{2}$ population with properties identical to the in vivo practical $F_{2}$ population data set, "default settings" (Jeuken and Lindhout 2002). The effects of five parameters on the starting-point $\mathrm{F}_{2}$ population are presented. Visually, the starting point is the middle point where all lines come together. Parameters of starting-point: $\mathrm{F}_{2}$ size $=126$, genotype frequency homozygous $L$. saligna $=0.25$, marker distance to $\mathrm{QTL}=5 \mathrm{cM}$, number of QTLs explaining the trait $=7$. For other parameters, see "Materials and methods." Genotype freq B means the genotype frequency of plants that are homozygous $L$. saligna at the specific QTL locus 
Table 2 Estimation of detection chance per QTL

\begin{tabular}{|c|c|c|c|c|c|c|c|}
\hline QTL & Detected in & $\begin{array}{l}\text { Allelic } \\
\text { effect }^{\mathrm{b}}\end{array}$ & $\begin{array}{l}\text { Average } \\
\text { marker } \\
\text { distance }^{c}\end{array}$ & $\begin{array}{l}\text { Segregation } \\
\text { ratio in } \mathrm{F}_{2}{ }^{\mathrm{d}}\end{array}$ & $\begin{array}{l}\text { Frequency } \\
\text { genotype } \\
\text { "homozygous } \\
\text { L. saligna" }\end{array}$ & $\begin{array}{l}\text { Detection } \\
\text { chance } \\
\text { in } F_{2}\end{array}$ & $\begin{array}{l}\text { Detection } \\
\text { chance } \\
\text { in BILs }\end{array}$ \\
\hline$R b q 1$ & $\mathrm{~F}_{2}+\mathrm{pBIL} 7.2$ & dom & 2.1 & $37: 44: 8$ & 0.09 & 0.91 & 0.98 \\
\hline $\begin{array}{l}R b q 3 / \\
r b q 3^{\mathrm{a}}\end{array}$ & $\mathrm{F}_{2}+$ BIL9.2 & $\mathrm{dom} / \mathrm{rec}$ & 2.7 & $29: 49: 9$ & 0.11 & $0.86 / 0.38$ & 0.98 \\
\hline$R b q 2$ & $\mathrm{~F}_{2}$ & dom & 2 & $1: 2: 1$ & 0.25 & 0.81 & 0.98 \\
\hline$r b q 7$ & BIL4.2 & rec & 2.5 & $1: 2: 1$ & 0.25 & 0.79 & 0.98 \\
\hline$r b q 5$ & BIL2.2 & rec & 3.9 & $1: 2: 1$ & 0.25 & 0.74 & 0.98 \\
\hline$r b q 6$ & BIL6.3 & $\mathrm{rec}$ & 3.3 & $36: 46: 9$ & 0.10 & 0.34 & 0.98 \\
\hline$r b q 4$ & BIL8.2 & rec & 2.3 & $30: 90: 6$ & 0.05 & 0.15 & 0.98 \\
\hline
\end{tabular}

a The inheritance of $r b q 3$ is still ambiguous

${ }^{\mathrm{b}}$ dom $=$ dominant, $\mathrm{rec}=$ recessive

c Average marker distance in QTL mapping region

${ }^{\mathrm{d}}$ The segregation ratio in $\mathrm{F}_{2}$ between genotypes $(a: h: b) ; a=$ homozygous $L$. sativa, $h=$ heterozygous, $b=$ homozygous $L$. saligna

e Note that the detection chance in BILs does not depend on any of the factors that vary between the $\mathrm{F}_{2}$-detected QTLs

indicates that there is still a small probability that a QTL, which was detected in an $F_{2}$, may go unnoticed in a BIL experiment. Our simulation program is a useful tool to quickly investigate these probabilities for given QTL effects and experimental conditions.

\section{Discussion}

Description of QTLs and their contribution

to non-host resistance

Seven QTLs have been detected in an $\mathrm{F}_{2}$ and a BIL strategy together: Rbq1, Rbq2, rbq3, rbq4, rbq5, rbq6 and $r b q 7$. Two QTLs are dominant, four QTLs are recessive and the inheritance for one QTL ( $r b q 3)$ is ambiguous. As the majority of the locations of the seven QTLs do not coincide with known $R$-gene clusters in the lettuce genome and the majority of the QTLs are recessive, these QTLs are not likely to be alleles of known $R$-genes or newly detected $R$ genes. Indications of candidate genes for the QTLs and their possible functions will be described elsewhere.

The effects of these seven single QTLs on resistance to B. lactucae are rather large, as each of them lowers the infection level to $31-76 \%$ of the infection severity of the susceptible parent line L. sativa Olof. Stacking two or three QTLs with an additive effect is probably already enough to reach $0 \%$ infection severity. Therefore, harboring seven QTLs seems redundant, but can also be an explanation for nonhost resistance: accumulation of several resistance layers by several QTLs with a rather strong effect and possibly present at high (favorable) allele frequencies.

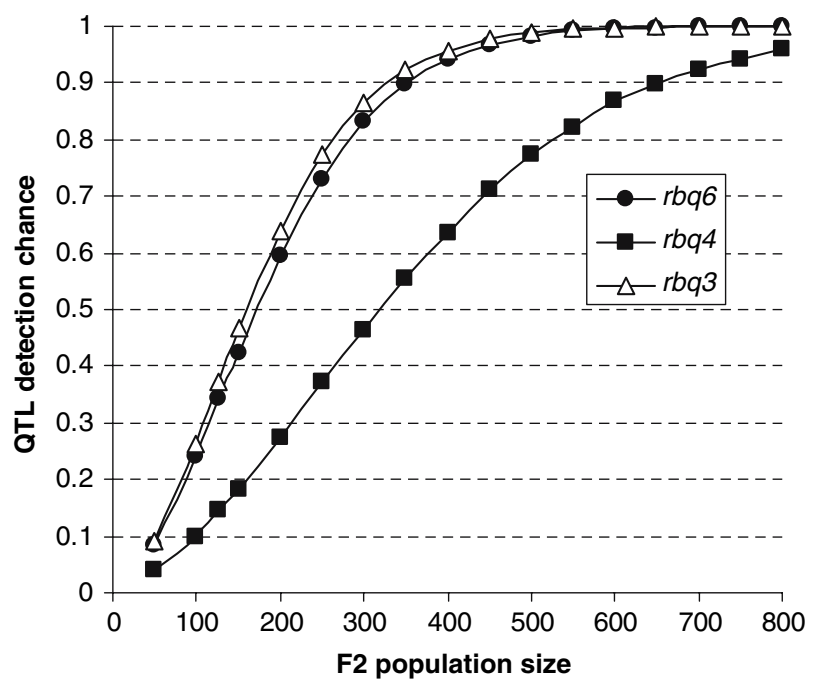

Fig. 6 The effect of $F_{2}$ population size on the QTL detection chances for recessive QTLs that are located in regions with severe distorted segregations. QTLs: $r b q 6=$ segregation ratio 36:46:9, average marker distance: 3.3. $r b q 4=$ segregation ratio 30:90:6, average marker distance: 2.3. $r b q 3=$ segregation ratio 29:49:9, average marker distance: 2.7

\section{Efficiency of QTL mapping}

\section{Detection of additional QTLs in a set of BILs}

The results between the six disease tests on the selections of BILs are very similar, despite the variations between the different experiments that were done over different seasons. This indicates that resistance governed 
by these QTLs is stable and rather insensitive to environmental conditions.

Six out of the set of 29 BILs showed a quantitative resistance to both $B$. lactucae races. One QTL is dominant, four QTLs are recessive and the inheritance for one QTL is ambiguous. The four recessive QTLs were mapped at new positions and the other two QTLs were mapped at positions detected earlier in the $\mathrm{F}_{2}$ population. The quantitative resistances lower the infection level to $31-76 \%$ of the infection severity level of the susceptible parent line L. sativa Olof. This is remarkable, as each of the three QTLs detected in the $\mathrm{F}_{2}$ population only showed an average reduction to $68 \%$. This confirms that QTL effects can be measured more precisely in BILs because of the increased accuracy of phenotyping and diminished genetic noise.

Although the heritability was high $(>0.8)$ and the environmental variance was low (0.3) in the $\mathrm{F}_{2}$ population, the three QTLs explained only $51 \%$ of the quantitative resistance, indicating that other resistance QTLs still were segregating in the $F_{2}$ population. The four newly detected QTLs in the BILs may represent QTLs that went unnoticed in the $\mathrm{F}_{2}$ population.

Skewness severely decreases detection chance of recessive QTLs in an $F_{2}$ population

Usually, it is impossible to verify the causes of the failure to identify QTLs in mapping studies. However, our data are very suitable to study the effect of the most important parameters on the power of QTL mapping. Therefore, we simulated QTL detection on $F_{2}$ populations and did a sensitivity analysis on the size of the effects of the most important parameters. By doing so, we showed that the parameters "population size" and "number of QTLs explaining the trait" had the largest effects, while the parameter "skewness" had only a large negative effect if the wild recessive resistance enhancing allele was underrepresented (deficit of $L$. saligna alleles, Fig. 5). This is not surprising, as in this case skewness will decrease the number of recessive homozygotes to far below the proportion of $25 \%$ (the average frequency in the case of no segregation distortion). With only a few plants in this marker class, a test for a difference between phenotypic means of marker genotype classes will lose much power. The same underrepresentation of wild alleles (deficit of $L$. saligna alleles) had a slight positive effect on detection chance of dominant QTLs. Taken together, the effects of direction of dominance and segregation distortion explain why we only detected dominant QTLs in the $F_{2}$ population (Table 2).
Backcross inbred lines are more efficient and powerful in QTL mapping than an $F_{2}$ population

In this study, the set of BILs reveals more QTLs for downy mildew resistance than the $\mathrm{F}_{2}$ population, and its detection chances for QTLs were higher than that in the $\mathrm{F}_{2}$ (Table 2). This is in agreement with several comparative studies between $\mathrm{AB}$ lines and classic segregating populations (like $\mathrm{BC}_{1}, \mathrm{~F}_{2}, \mathrm{DH}$ and RILs) in tomato for fruit quality traits (Grandillo and Tanksley 1996; Tanksley et al. 1996) and in barley for agronomic and yield quality traits (Pillen et al. 2004). In a comparative study between NILs and RILs in Arabidopsis for developmental traits, similar numbers of total QTLs were detected between the two populations, although different loci were detected (Keurentjes et al. 2007). Unique QTLs that showed epistatic interactions were detected in the RILs, and unique small-effect QTLs were detected in the NILs. A comparative study between AB lines and RILs in barley for seedling resistance traits showed the same number of QTLs (Yun et al. 2006). Generalizing, we may state that Inbred Line strategies most of the time reveal more QTLs than classical population studies, which is in agreement with theoretical studies (Tanksley et al. 1996).

Most comparative studies cannot address specific reasons for this difference in QTL detection. Based on our simulation study, we can, for two out of four newly detected QTLs, address a reason for being unnoticed in the $\mathrm{F}_{2}$ : the combination of recessiveness of the trait with skewness against the recessive wild species alleles. A similar reason was suggested for detection of a heading date QTL in a BIL and being unnoticed in the $\mathrm{F}_{2}$ in a study in rice (Lin et al. 1998). Skewness occurs in many segregation populations, which suggests that in QTL mapping experiments on relatively small segregating populations $(N<200)$, several QTLs may remain undetected. The abundance and degree of skewness in a genetic mapping population is thought to be associated with the level of genomic divergence between parental species (Jenczewski et al. 1997; Taylor and Ingvarsson 2003; Truco et al. 2007). This indicates that in QTL mapping studies, the size of the $\mathrm{F}_{2}$ or RIL population should increase as the genetic distance between the parents gets larger. This supports the choice for the development of a set of BILs in wide crosses. A set of BILs has more power to detect, especially recessive QTLs, because of its advantageous segregation ratio compared to $F_{2}$ or RILs.

The reason why two QTLs that were identified in the BILs were not detected in the $\mathrm{F}_{2}$ is less obvious. Theoretically, there are three possible causes: (a) a negative trans-acting epistatic interaction, (b) the magnitude of the effect is too small to raise the LOD value above the set threshold and (c) just "bad luck." 
By its very nature, epistasis of the type mentioned earlier cannot be removed by experimental set up nor can it be detected by means of a special statistical analysis. Therefore, some QTLs may always be hidden in the "genetic background noise." Reasons (b) and (c) mentioned earlier are, of course, not mutually exclusive. With an a posteriori detection probability of around $70 \%$ (cf. Table 2), the chance to miss two QTLs is 0.09 . On one hand this may be considered "bad luck," but on the other hand this is not extremely unlikely. The reason why one QTL was detected in the $\mathrm{F}_{2}$ only, and not in the BILs, might theoretically be explained by a positive trans-acting epistatic interaction in the $F_{2}$. This study clearly shows the added value of extended genetic studies on two types of populations (BILs and $\mathrm{F}_{2}$ ) instead of one type only in dissecting complex genetic traits.

The function of these QTLs in nonhost resistance is still completely obscure and awaits further detailed molecular phytopathological studies. This will contribute to our knowledge of nonhost resistance and how it may have evolved during evolution. For the time being, plant breeders can already use these QTLs in their continuous fight to control downy mildew in lettuce.

Acknowledgments This research is supported by the Dutch Technology Foundation STW, Applied Science Division of NWO and the Technology Program of the Ministry of Economic Affairs. The EST data was obtained from The Compositae Genome Project website at compgenomics.ucdavis.edu that was supported by the USDA IFAFS and the NSF Plant Genome programs.

Open Access This article is distributed under the terms of the Creative Commons Attribution Noncommercial License which permits any noncommercial use, distribution, and reproduction in any medium, provided the original author(s) and source are credited.

\section{Appendix A}

The simulation program to carry out in silico QTL detection experiments was written to answer the following question: What is the probability that a QTL of given effect will be detected by means of a statistical test in an $F_{2}$ population or in a set of BILs? To answer this question one has to assume (or to know) the following quantities:

- The genetic variance caused by other QTLs that affect the trait

- The size of the $\mathrm{F}_{2}$ population and the number of BILs

- The environmental (or error) variance per observation

- The number of observations per $F_{2}$ or BIL plant

- The number of plants per BIL and the number of plants per control genotype
Simulation of $\mathrm{F}_{2}$ experiments

Since QTL detection is based on the notion that a marker closely linked to a QTL will "explain" part of the variance caused by that QTL, one also needs to know the genetic distance between the QTL and the nearest marker. Since we also wished to account for skewed segregation ratios at marker loci (and nearby QTLs), we decided to simulate the QTL mapping procedure rather than following an analytic approach to the question of detection power. Table 3 presents the parameters that are required to run a simulation. Using the parameters mentioned in Table 3, we first partition the variance as follows, using standard results from quantitative genetics.

The additive and dominance effects at the QTL read

$a=\frac{1}{2}\left(\mu_{Q Q}-\mu_{q q}\right)$

and

$d=\mu_{Q q}-\frac{1}{2}\left(\mu_{Q Q}+\mu_{q q}\right)$,

respectively.

This results in a genotypic variance caused by this single QTL of

$V_{\mathrm{G}(\mathrm{QTL})}=\frac{1}{2} a^{2}+\frac{1}{4} d^{2}$.

Since we have $n$ QTL of this same effect, the total genetic variance in the $\mathrm{F}_{2}$ equals

$V_{\mathrm{G}(\mathrm{tot})}=n V_{\mathrm{G}(\mathrm{QTL})}$.

The genetic background variance caused by the $(n-1)$ remaining QTLs equals

$V_{\mathrm{G}(\mathrm{rest})}=\left(1-\frac{1}{n}\right) V_{\mathrm{G}(\mathrm{tot})}=(n-1) V_{\mathrm{G}(\mathrm{QTL})}$.

Thus, the nongenetic (or error) variance equals

Table 3 Parameters that are required to run a simulation

\begin{tabular}{ll}
\hline Symbol & Description \\
\hline $\begin{array}{l}\mu_{Q Q}, \mu_{Q q}, \\
\mu_{q q}\end{array}$ & Genotypic values of QTL genotypes \\
$n$ & Number of segregating QTLs \\
$V_{\mathrm{e}}$ & Error variance per observation \\
$k$ & Number of observations per plant \\
$r$ & Recombination frequency between QTL and nearest \\
& marker \\
$V_{\mathrm{P}}$ & Total phenotypic variance in the $\mathrm{F}_{2}$ population \\
$f_{M M}, f_{M m}$, & Frequencies at marker locus (possibly skewed) \\
$f_{m m}$ & \\
\hline
\end{tabular}


$V_{\mathrm{e}}=V_{\mathrm{P}}-V_{\mathrm{G}(\mathrm{tot})}$,

and the error variance attached to the mean of $k$ observations per plant reads

$V_{\mathrm{E}}=\frac{V_{\mathrm{e}}}{k}$.

In the procedure, described step-by-step below, we first sample marker genotypes and then, depending on the marker genotype ( $M M, M m$ or $m m$ ), we sample the QTL genotype $(Q Q, Q q$ or $q q)$. These conditional probabilities read

$$
\begin{aligned}
P(Q Q \mid M M) & =(1-r)^{2} \\
P(Q q \mid M M) & =2 r(1-r) \\
P(q q \mid M M) & =r^{2} \\
P(Q Q \mid M m) & =r(1-r) \\
P(Q q \mid M m) & =1-2 r(1-r) \\
P(q q \mid M m) & =r(1-r) \\
P(Q Q \mid m m) & =r^{2} \\
P(Q q \mid m m) & =2 r(1-r) \\
P(q q \mid m m) & =(1-r)^{2} .
\end{aligned}
$$

(Notice that the above implies that in the parents the alleles $M$ and $Q$ occur together, as well as $m$ and $q$ ).

Marker genotypes are sampled from the given segregation ratio. For each individual sampled, the marker genotype as well as its phenotypic score is recorded. After having sampled the required number of $F_{2}$ plants, an analysis of variance is performed, and a test statistic is calculated to test whether or not the differences between the means of the marker classes are significant. To correct for multiple testing (that is to control the type 2 error), we have set the significance threshold corresponding to $P=0.005$ (rather than 0.05 ).

In the following, $N\left(\mu, \sigma^{2}\right)$ represents a sample from a normal distribution with mean $\mu$ and variance $\sigma^{2}$.

A single simulation "case" comprises the following steps:

1. Sample a marker genotype from the distribution $\left\{f_{M M}, f_{M m}, f_{m m}\right\}$ (result is $M M, M m$ or $m m$ ).

2. Sample a QTL genotype, conditional on the marker genotype (result is $Q Q, Q q$ or $q q$ ).

3. Set genotypic value (result is $\mathrm{gv}=\mu_{Q Q}, \mu_{Q q}, \mu_{q q}$, according to the result at step 2).

4. Add a random term to gv, representing the joint effect of the other QTLs: $G=\mathrm{gv}+\left(0, V_{\mathrm{G}(\mathrm{rest})}\right)$.

5. Add a random term to obtain the phenotypic score (average of $k$ observations): $y=G+N\left(0, V_{E}\right)$.

6. Store marker genotype and phenotypic score.

7. Repeat steps $1-6 N$ times $(N=$ population size).

8. Test significance between scores of marker genotypic classes.
9. Store outcome of test.

10. Repeat steps 1-9 50,000 times.

11. Calculate relative frequency of significant test results.

Simulation of BIL experiment

In simulating a BIL experiment, we proceed as follows. Using the same genotypic values for the homozygous $L$. sativa and $L$. saligna genotypes and the appropriate environmental variances, two series of phenotypic values, representing the BIL and control observations, are generated. Note that these two samples are not necessarily of the same size. The difference between the two sample means is tested by a two-sample $t$ test, using a Bonferroni correction for multiple testing to set the required $P$-value (the number independent test being equal to the number of BILs being tested). This procedure is repeated 50,000 times (or as many as the user judges appropriate) and the proportion of significant tests is taken as the detection probability.

\section{Discussion}

In our simulations, we have used an analysis of variance (ANOVA) per marker rather than the widely used methods of interval mapping (Lander and Botstein 1989) or composite interval mapping (Jansen and Stam 1994; Zeng 1994). Although the latter are known to be more powerful than ANOVA at single markers, the difference in power between the two approaches rapidly decreases as marker density on the linkage map increases.

A more important and more practical reason to use the ANOVA approach in the simulations is its relative simplicity as compared to the maximum likelihood procedure of interval mapping. This simplicity also enabled us to provide an easy-to-use simulation program that is available to the scientific and plant breeding community, enabling a very quick exploration of the statistical power of QTL detection in $F_{2}$ populations and sets of BILs with various settings of the factors involved.

The program, powQTL, was written in $\mathrm{C}++$; an executable is available from P.S. upon request.

\section{References}

Arcade A, Labourdette A, Falque M, Mangin B, Chardon F, Charcosset A, Joets J (2004) BioMercator: integrating genetic maps and QTL towards discovery of candidate genes. Bioinformatics 20:2324-2326

Beavis WD (1994) The power and deceit of QTL experiments: lessons from comparative QTL studies. In: 49th Annual Corn 
and Sorghum Industry Research Conference. ASTA, Washington, DC, pp 250-266

Beavis WD (1998) QTL analyses: power, precision and accuracy. In: Paterson AH (ed) Molecular dissection of complex traits. CRC Press, Boca Raton, pp 145-162

Flint-Garcia SA, Thornsberry JM, Buckler ES IV (2003) Structure of linkage disequilibrium in plants. Annu Rev Plant Biol 54:357374

Grandillo S, Tanksley SD (1996) QTL analysis of horticultural traits differentiating the cultivated tomato from the closely related species Lycopersicon pimpinellifolium. Theor Appl Genet 92:935-951

Guo B, Sleper DA, Nguyen HT, Arelli PR, Shannon JG (2006) Quantitative trait loci underlying resistance to three soybean cyst nematode populations in soybean PI 404198A. Crop Sci 46:224233

Gupta PK, Rustgi S, Kulwal PL (2005) Linkage disequilibrium and association studies in higher plants: present status and future prospects. Plant Mol Biol 57:461-485

Jansen RC, Stam P (1994) High resolution of quantitative traits into multiple loci via interval mapping. Genetics 136:1447-1455

Jenczewski E, Gherardi M, Bonnin I, Prosperi JM, Olivieri I, Huguet $T$ (1997) Insight on segregation distortions in two intraspecific crosses between annual species of Medicago (Leguminosae). Theor Appl Genet 94:682-691

Jeuken M, Lindhout P (2002) Lactuca saligna, a non-host for lettuce downy mildew (Bremia lactucae), harbors a new race-specific Dm gene and three QTLs for resistance. Theor Appl Genet 105:384-391

Jeuken M, van Wijk R, Peleman J, Lindhout P (2001) An integrated interspecific AFLP map of lettuce (Lactuca) based on two $L$. sativa $\times L$. saligna $\mathrm{F}-2$ populations. Theor Appl Genet 103:638-647

Jeuken MJW, Lindhout P (2004) The development of lettuce backcross inbred lines (BILs) for exploitation of the Lactuca saligna (wild lettuce) germplasm. Theor Appl Genet 109:394401

Kesseli RV, Paran I, Michelmore RW (1994) Analysis of a detailed genetic linkage map of Lactuca sativa (Lettuce) constructed from RFLP and RAPD markers. Genetics 136:1435-1446

Keurentjes JJB, Bentsink L, Alonso-Blanco C, Hanhart CJ, Blankestijn-De Vries H, Effgen S, Vreugdenhil D, Koornneef M (2007) Development of a near-isogenic line population of Arabidopsis thaliana and comparison of mapping power with a recombinant inbred line population. Genetics 175:891-905

Kraakman ATW, Niks RE, Van den Berg PMMM, Stam P, Van Eeuwijk FA (2004) Linkage disequilibrium mapping of yield and yield stability in modern spring barley cultivars. Genetics 168:435-446

Lander ES, Botstein D (1989) Mapping mendelian factors underlying quantitative traits using RFLP linkage maps. Genetics 121:185199

Lin SY, Sasaki T, Yano M (1998) Mapping quantitative trait loci controlling seed dormancy and heading date in rice, Oryza sativa L., using backcross inbred lines. Theor Appl Genet 96:997-1003

Lindhout P (2002) The perspectives of polygenic resistance in breeding for durable disease resistance. Euphytica 124:217226
Patterson HD, Thompson R (1971) Recovery of inter-block information when block sizes are unequal. Biometrika 58:545-554

Peleman JD, Wye C, Zethof J, Sorensen AP, Verbakel H, van Oeveren J, Gerats T, van der Voort JR (2005) Quantitative trait locus (QTL) isogenic recombinant analysis: a method for highresolution mapping of QTL within a single population. Genetics 171:1341-1352

Pillen K, Zacharias A, Léon J (2004) Comparative AB-QTL analysis in barley using a single exotic donor of Hordeum vulgare ssp. spontaneum. Theor Appl Genet 108:1591-1601

Schön CC, Utz HF, Groh S, Truberg B, Openshaw S, Melchinger AE (2004) Quantitative trait locus mapping based on resampling in a vast maize testcross experiment and its relevance to quantitative genetics for complex traits. Genetics 167:485-498

Tanksley SD, Grandillo S, Fulton TM, Zamir D, Eshed Y, Petiard V, Lopez J, Beck-Bunn T (1996) Advanced backcross QTL analysis in a cross between an elite processing line of tomato and its wild relative L. pimpinellifolium. Theor Appl Genet 92:213-224

Tanksley SD, Nelson JC (1996) Advanced backcross QTL analysis: a method for the simultaneous discovery and transfer of valuable QTLs from unadapted germplasm into elite breeding lines. Theor Appl Genet 92:191-203

Taylor DR, Ingvarsson PK (2003) Common features of segregation distortion in plants and animals. Genetica 117:27-35

Truco M, Antonise R, Lavelle D, Ochoa O, Kozik A, Witsenboer H, Fort S, Jeuken M, Kesseli R, Lindhout P, Michelmore R, Peleman J (2007) A high-density, integrated genetic linkage map of lettuce (Lactuca spp.). Theor Appl Genet 115:735-746

Vales MI, Schön CC, Capettini F, Chen XM, Corey AE, Mather DE, Mundt CC, Richardson KL, Sandoval-Islas JS, Utz HF, Hayes PM (2005) Effect of population size on the estimation of QTL: a test using resistance to barley stripe rust. Theor Appl Genet 111:1260-1270

Van de Wiel C, Arens P, Vosman B (1999) Microsatellite retrieval in lettuce (Lactuca sativa L.). Genome 42:139-149

Van Ooijen JW (1999) LOD significance thresholds for QTL analysis in experimental populations of diploid species. Heredity 83:613624

Van Ooijen JW (2004) MapQTL® 5: software for the mapping of quantitative trait loci in experimental populations. Kyazma B.V., Wageningen, Netherlands

Varshney RK, Hoisington DA, Tyagi AK (2006) Advances in cereal genomics and applications in crop breeding. Trends Biotechnol 24:490-499

Yu J, Pressoir G, Briggs WH, Vroh Bi I, Yamasaki M, Doebley JF, McMullen MD, Gaut BS, Nielsen DM, Holland JB, Kresovich S, Buckler ES (2006) A unified mixed-model method for association mapping that accounts for multiple levels of relatedness. Nat Genet 38:203-208

Yun SJ, Gyenis L, Bossolini E, Hayes PM, Matus I, Smith KP, Steffenson BJ, Tuberosa R, Muehlbauer GJ (2006) Validation of quantitative trait loci for multiple disease resistance in barley using advanced backcross lines developed with a wild barley. Crop Sci 46:1179-1186

Zamir D (2001) Improving plant breeding with exotic genetic libraries. Nat Rev Genet 2:983-989

Zeng ZB (1994) Precision mapping of quantitative trait loci. Genetics 136:1457-1468 